\title{
Bilateral Branchial Arch Anomalies: A Rare Case Report
}

\section{Pradeep P*, Swagatika S, Dillip KS and Pradipta KP \\ Department of Otorhinolaryngology, All India Institute of Medical Sciences, India}

*Corresponding author: Pradeep Pradhan, MS (ENT), Department of Otorhinolaryngology, All India Institute of Medical Sciences, Bhubaneswar, Odisha, India, Pin-751019, Tel: +91 9968634053; E-mail: padiapradhan@gmail.com

\section{Case Report \\ Volume 2 Issue 2}

Received Date: July 19, 2017

Published Date: September 11, 2017

DOI: $10.23880 /$ ooaj-16000154

\section{Abstract}

Branchial sinuses/fistula is one of the most common congenital anomalies found in neck derived from the branchial arches. Although unilateral lesions are common, very rarely it can be bilateral and patient can present as discharging sinus/fistula over the anterior aspect of the neck. Here, we have presented a rare case report of bilateral branchial arch anomaly from the second branchial arch without any syndromic association.

Keywords: Branchial fistula/sinus; Second arch

\section{Introduction}

Branchial arch anomalies are the developmental defect of branchial apparatus [1]. Second branchial anomalies are the commonest, accounting $90 \%$ of all the disorder $[2,3]$. However the bilateral occurrence of the branchial arch anomalies is very uncommon $(2-3 \%)$ in the routine clinical practice [4]. Again the simultaneous presence of both branchial fistulae and a branchial sinus from second arch is even rarer. Here we have focused a case of bilateral branchial arch anomaly presented with a sinus in left and a fistula in the right side of the neck, managed successfully by surgical excision.

\section{Case Report}

A 22-year-old female presented to outpatient department with bilateral pinpoint openings at the lower one-third of lateral neck. The patient reported these openings since birth, with history of intermittent fluid discharge. The amount of discharge increases during episodes of upper respiratory tract infections and subside with antibiotic treatment particularly in the right side. The pinna was normal in shape, size and position, and the tympanic membrane was normal. Bilateral fistulography indicated (Figures $1 \& 2$ ) the presence of complete branchial tracts with spillage of contrast material in tonsillar fossa in the right side and a blind sac on the left side respectively. Ultrasonography of the abdomen and renal examination revealed normal kidney function, ruling out the possibility of branchio-oto-renal syndrome. Both the fistula and sinus were excised under general anaesthesia and the final specimens were sent for histopathological examination and the final diagnosis of branchial sinus/fistula was confirmed (Figure 3). The patient's postoperative course of the patient was found to be uneventful, and she is asymptomatic for the past 6 months without recurrence of symptoms.

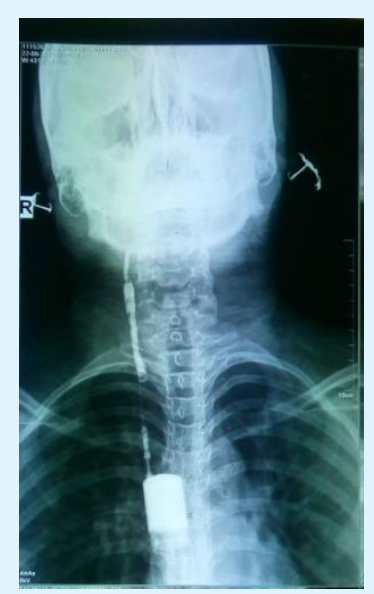

Figure 1: Contrast $\mathrm{X}$ ray of the neck shows a fistulous tract in the right side of the neck opening in the right tonsillar fossa. 


\section{Otolaryngology Open Access Journal}

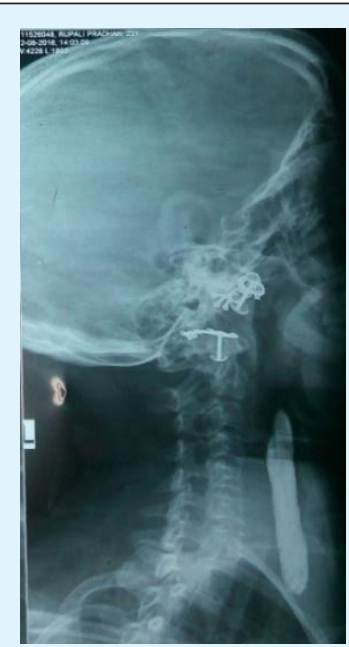

Figure 2: Contrast $\mathrm{X}$ ray demonstrates a sinus in the left side of the neck.

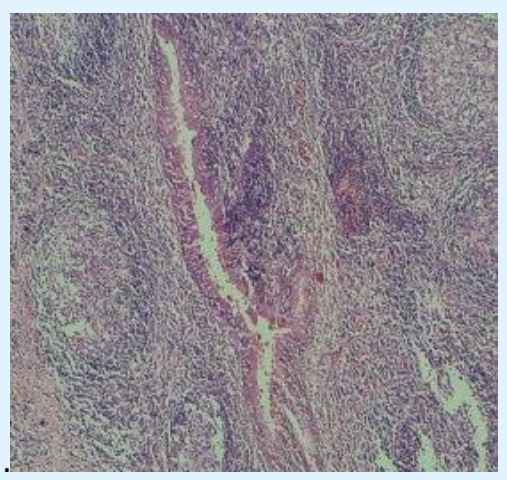

Figure 3: Histopathological photo shows fistulous tract lined by ciliated stratified columnar epithelium, subepithelium shows lymphoid follicles with germinal center. (H\&E, X40)

\section{Discussion}

Branchial arch anomalies are one of the commonest congenital defects seen in the paediatric age group and was first described by Baer in 1827. It can be presented as a cyst, sinus or a fistula in the anterior-lateral aspect of the neck in front of the sternocleidomastoid muscle. Usually the branchial cyst presents with an external swelling in the neck without any internal/external opening. In contrast, branchial sinus will have an external opening and fistula will have both external and internal opening in the neck. Cysts are considered to be the remnant of the branchial cleft or pouch which later can present with branchial sinus or fistula. The first branchial arch is related to the auricle and can appear as an epidermoid element can travel close to the facial nerve. Anomaly from the second arch is the commonest among all, can present as cyst, sinus or fistula. External opening lies anterior to the sternocleidomastoid mastoid muscle and the internal opening varies according to the arch of origin. When it originates from the second arch, fistulous tract traverse deep to the second arch structures, including the external carotid artery, the stylohyoid muscle, and the posterior belly of the digastric muscle to open in the tonsillar fossa. Rarely, it opens to the pyriform sinus where the fistulous tract, travels deep to glossopharyngeal nerve and the internal carotid artery. Fourth arch derivatives are extremely rare in clinical practice. Occurrence of bilateral branchial sinus/fistula is very rare. It occurs in $25 \%-50 \%$ of cases and are more likely to be hereditary (10). This is unlike in our case where there was no family history of such association. About $96 \%-97 \%$ of these anomalies are unilateral and only $2 \%-3 \%$ have a bilateral presentation and the rate of bilateralism is higher in familial cases [4]. A review of the English literature found only six cases of bilateral branchial cleft fistulas [5-8]. In all the previously reported cases of bilateral cleft anomalies, the patients had a family history of such anomalies. Similar findings have also been observed by Gupta et al in their case report [9]. Surgical excision is the only treatment offered to the symptomatic patients with branchial arch disorder. Antibiotics are advised whenever it is infected and in the post-operative period. Of the five reported cases of bilateral branchial sinus, Gatti \& Zimm [6] reported two cases, [6] which were managed with surgical excision. Stair-step or stepladder incision usually recommended for complete excision of the tract as was performed in our case. Although surgical excision is not difficult, it needs a clear understanding of branchial arch and meticulous dissection to preserve the vital structures for a better clinical outcome.

\section{Conclusion}

In conclusion, simultaneous presentation of branchial sinus and fistula from the second arch is very rare. Detail anatomical knowledge and meticulous dissection of the disorder is inevitable to prevent the complications and recurrence.

Compliance with Ethical Standards Disclosure of potential conflicts of interest: There is no conflict of interest among the authors and this research has received no financial grant from any funding agency, commercial or not-for-profit sectors.

Ethical approval: All procedures performed in studies involving human participants were in accordance with the ethical standards of the institution research committee 


\section{Otolaryngology Open Access Journal}

and with the 1964 Helsinki declaration and its later amendments or comparable ethical standards.

Informed consent: Informed consent was obtained from all individual participants included in the study.

\section{References}

1. Alber GD (1963) Branchial anomalies. JAMA 399-409.

2. Olsen KD, Maragos NE, Weiland LH (1980) First branchial cleft anomalies. Laryngoscope 90(3): 423436.

3. Jaka RC, Singh G (2007) Complete congenital third brachial fistula on right side. Otolaryngol Head Neck Surg 137(3): 518-519.

4. Chandler JR, Mitchell B (1981) Branchial cleft cysts, sinuses, and fistulas. Otolaryngol Clin North Am 14(1): 175-186.
5. Schewitsch I, Stalsberg H, Schroder KE, Mair IW (1980) Cysts and sinuses of the lateral head and neck. J Otolaryngol 9(1): 1-6.

6. Gatti WM, Zimm J (1988) Bilateral branchial cleft fistulas: diagnosis and management of two cases. Ear Nose Throat J 67: 256, 258, 261.

7. Gold BM (1980) Second branchial cleft cyst and fistula. AJR Am J Roentgenol 134: 1067-1069.

8. Shvero J, Hadar T, Avidor I, Abraham A, Sidi J (1986) Heterotopic salivary tissue and branchial sinuses. J Laryngol Otol 100(2): 243-246.

9. Gupta AK, Kumar S, Jain A (2008) Bilateral first and second branchial cleft fistulas: a case report. Ear Nose Throat J 87(5): 291-293. 